\title{
Binary neutron star mergers and third generation detectors: Localization and early warning
}

\author{
Man Leong Chan," Chris Messenger, Ik Siong Heng, and Martin Hendry \\ SUPA, School of Physics and Astronomy, University of Glasgow, Glasgow G12 8QQ, United Kingdom
}

(Received 6 March 2018; published 28 June 2018)

\begin{abstract}
For third generation gravitational wave detectors, such as the Einstein Telescope, gravitational wave signals from binary neutron stars can last up to a few days before the neutron stars merge. To estimate the measurement uncertainties of key signal parameters, we develop a Fisher matrix approach which accounts for effects on such long duration signals of the time-dependent detector response and the Earth's rotation. We use this approach to characterize the sky localization uncertainty for gravitational waves from binary neutron stars at 40, 200, 400, 800, and $1600 \mathrm{Mpc}$, for the Einstein Telescope and Cosmic Explorer individually and operating as a network. We find that the Einstein Telescope alone can localize the majority of detectable binary neutron stars at a distance of $\leq 200 \mathrm{Mpc}$ to within $100 \mathrm{deg}^{2}$ with $90 \%$ confidence. A network consisting of the Einstein Telescope and Cosmic Explorer can enhance the sky localization performance significantly - with the $90 \%$ credible region of $\mathcal{O}(1) \mathrm{deg}^{2}$ for most sources at $\leq 200 \mathrm{Mpc}$ and $\leq 100 \mathrm{deg}^{2}$ for most sources at $\leq 1600 \mathrm{Mpc}$. We also investigate the prospects for third generation detectors identifying the presence of a signal prior to merger. To do this, we require a signal to have a network signalto-noise ratio of $\geq 12$ and $\geq 5.5$ for at least two interferometers, and to have a $90 \%$ credible region for the sky localization that is no larger than $100 \mathrm{deg}^{2}$. We find that the Einstein Telescope can send out such "earlywarning" detection alerts 1-20 hours before merger for $100 \%$ of detectable binary neutron stars at $40 \mathrm{Mpc}$ and for $\sim 58 \%$ of sources at $200 \mathrm{Mpc}$. For sources at a distance of $400 \mathrm{Mpc}$, a network of the Einstein telescope and Cosmic Explorer can produce detection alerts up to $\sim 3$ hours prior to merger for $98 \%$ of detectable binary neutron stars.
\end{abstract}

DOI: $10.1103 /$ PhysRevD.97.123014

\section{INTRODUCTION}

The first detections of gravitational waves (GWs) from binary black hole (BBH) systems GW150914, GW151226, GW170104, and GW170608 by the two LIGO detectors at Hanford and Livingston [1-4] have opened a new window on the universe and marked the beginning of gravitational wave (GW) astronomy. In 2017, VIRGO began observation and the first joint detection GW170814 was made by LIGO and VIRGO together [5]. Just a few days later, the three GW observatories detected the first binary neutron star (BNS) merger event GW170817 [6]. The detections of multiple electromagnetic (EM) counterparts associated with GW170817 initiated the era of GW multi-messenger astronomy [7-11]. In the coming years, additional groundbased interferometric detectors such as KAGRA and LIGO India are likely to join the global network [12-14]. Many

\footnotetext{
"m.chan.1@ research.gla.ac.uk
}

Published by the American Physical Society under the terms of the Creative Commons Attribution 4.0 International license. Further distribution of this work must maintain attribution to the author(s) and the published article's title, journal citation, and DOI. more GW detections can therefore be expected, from several different GW sources in the universe such as compact binary mergers [i.e., BBH, BNS and neutron star black hole (NSBH)], core-collapse supernovae, nonsymmetric neutron stars, and the stochastic background $[15,16]$.

For systems such as BNSs and NSBHs, the presence of a neutron star component is expected to lead to the generation of associated EM emission accompanying the mergers of these systems-including gamma ray bursts, $\mathrm{x}$-ray emission, kilonovae in the optical and infrared bands and radio afterglows [17-20]. Detecting an EM counterpart in coincidence with a GW trigger will increase the detection confidence and improve the sky localization of the GW detection. In addition, a successful EM follow-up observation can establish an association between the GW trigger and its progenitor and provide a better understanding of the progenitor and its local environment [21-23]. A coincident detection of an EM counterpart may also allow measurement of the redshift of the source independently of the GW signal; this can be used for cosmological tests and for constraining the equation of state of dark energy [24-26]. Despite the low probability that binary black hole (BBH) mergers will be accompanied by EM emission, identifying 
the host galaxy of a BBH will still hopefully establish the association between the $\mathrm{BBH}$ and the galaxy.

One key factor to the success of EM follow-up observations triggered by $\mathrm{GW}$ events, or that of identifying the host galaxy, is the localization of the GW source. However, GW interferometric detectors are not directional instruments, and usually triangulation from a network of GW detectors is the approach to localizing GW sources. Therefore, their performances are usually relatively poor when it comes to pinpointing sources of GWs. For example, the $90 \%$ credible region for the sky positions of the first detected BBH events are $\mathcal{O}\left(10^{2}\right) \operatorname{deg}^{2}$ [1,2]. The situation improved with VIRGO joining the observing runs, and it will continue to improve when KAGRA and LIGO India start operating in later years [12,13,27-31]. However, for sources at large distances, EM follow-up observations will remain a difficult challenge. This is because a given type of GW source observed at a larger distance will also have a larger associated localization error on the sky. Larger distances also mean that the telescopes performing EM follow-up observations will need to observe for longer to reach the sensitivity required to detect the counterparts [29]. As a result, the telescopes may be unable to scan the whole $90 \%$ credible region before the EM counterpart fades below the detectable threshold. For example, the brightness of kilonovae may peak at 1 day after the merger and then start fading [18] as was the case for the kilonova associated with GW170817 [11].

Third generation detectors such as the Einstein Telescope (ET) and Cosmic Explorer (CE) can bring significant improvements in GW source localization. Third generation detectors with enhanced sensitivity across the frequency band accessible to ground-based detectors will be able to detect GWs from $\mathrm{BBH}$ and BNS sources located at distances far beyond the horizon of second generation detectors. In particular, the improved sensitivity from 1 to $10 \mathrm{~Hz}$ of these detectors will distinguish them from second generation detectors, as this allows for an extended duration of in-band observation of the signal. Depending on the distance, signals from BNS can be traced back up to hours or days before merger.

The long in-band duration of a signal will introduce several effects. Firstly, it allows the detector to accumulate signal to noise ratio (SNR) over a significantly longer period of time. As mentioned, one of the difficulties in EM follow-up observations of BNS merger triggers at large distance will be the time available to scan the $90 \%$ credible region associated with the BNS trigger before the EM counterpart becomes too faint. If the SNR of a BNS trigger can be accumulated to a statistically significant level prior to the merger, prompt detection alerts could be made possible - thus increasing the probability of detecting the EM signature of the trigger [32]. The long duration also enables the detector to observe the source from different positions and directions as the earth rotates. This effect is important in localizing the source of GWs as it result in the time-dependency of the antenna pattern. The GW will also be Doppler shifted as the detector moves relative to the source as it rotates with the Earth's spin. The long duration of a signal thus requires the consideration of the earth's rotation when estimating the localization error.

In the literature, most studies of localization errors of GWs from compact binary coalescences are based on the assumption that the duration of the signal is short enough that the rotation of the earth is negligible $[12,27,33]$. This is justifiable for the reason that the in-band durations of the signals in those studies are only seconds to minutes in length. Mills, et al., 2017 [34] consider localization of short transient signals from BNS mergers with a network of both second and three generation detectors. More recently, there has been work considering the long inband duration and the rotation of the earth [35]. In this latter work the authors have modeled the GW signal using the stationary phase approximation-essentially the leading-order term in an expansion in powers of the small quantity that is the ratio of the radiation-reaction timescale and the orbital period.

In this work, we take into account the Earth's rotation and, using a Fisher matrix approach, estimate the localization of GWs from BNS sources observed by the ET and CE individually and as a network. We perform a series of tests to estimate the localization capabilities of these detectors for BNSs at 40, 200, 400, 800, and $1600 \mathrm{Mpc}$ and for a population of BNS sources that are distributed uniformly in the comoving volume. We then investigate the feasibility of "early-warning" detection by setting requirements on localization error and accumulated SNR before merger for an alert to be released. Throughout this work, we focus our analysis on BNSs where the in-band duration of their signals can be days' long, and thus the effect of Earth's rotation is important.

This paper is structured as follows: in Sec. II, we briefly introduce the configuration of the detectors we considered for this work, and the technologies employed to achieve their sensitivities. The methodology is presented in Sec. III with the results and simulations presented in Sec. IV together with a discussion. We then provide our conclusions in Sec. V.

\section{THIRD GENERATION DETECTORS}

There are currently two proposed third generation detectors: the ET and CE. For the ET, we employed the geometrical configuration known as ET-D as discussed in [36] where the detector consists of 3 individual interferometers. Each interferometer has an opening angle equal to $60^{\circ}$ and is rotated by $120^{\circ}$ relative to the others, thus forming an equilateral triangle. The lengths of the arms of the interferometers are $10 \mathrm{~km}$. The current design for CE is an interferometric detector with a $90^{\circ}$ opening angle and the arms as long as $40 \mathrm{~km}$ [37]. 


\section{A. Location and antenna pattern}

The exact geographic locations at which the detectors will be built are still unknown. In this work, the location adopted for the ET is (Longitude, Latitude) = $\left(10.4^{\circ}, 43.7^{\circ}\right)$, and for $\mathrm{CE}$ is $\left(-119.41^{\circ}, 46.45^{\circ}\right)$. Due to the differences between the ET and CE configurations, the antenna patterns of these detectors will be computed differently. The antenna pattern of each interferometer of the ET can be expressed as [38]

$$
\begin{gathered}
F_{+}^{1}(\theta, \phi, \psi)=-\frac{\sqrt{3}}{4}\left[\left(1+\cos ^{2} \theta\right) \sin 2 \phi \cos 2 \psi\right. \\
+2 \cos \theta \cos 2 \phi \sin 2 \psi], \\
F_{\times}^{1}(\theta, \phi, \psi)=\frac{\sqrt{3}}{4}\left[\left(1+\cos ^{2} \theta\right) \sin 2 \phi \sin 2 \psi\right. \\
-2 \cos \theta \cos 2 \phi \cos 2 \psi], \\
F_{+, \times}^{2}(\theta, \phi, \psi)=F_{+, \times}^{1}\left(\theta, \phi+\frac{2 \pi}{3}, \psi\right), \\
F_{+, \times}^{3}(\theta, \phi, \psi)=F_{+, \times}^{1}\left(\theta, \phi-\frac{2 \pi}{3}, \psi\right),
\end{gathered}
$$

where the above equations assume that the detector is at the center of a spherical coordinate system in which $\theta$ and $\phi$ are the azimuthal angle and polar angle of the source respectively, and $\psi$ is the GW polarization angle. The superscripts indicate the 3 interferometers in the configuration.

A single interferometer with arms separated by $60^{\circ}$ will have a response smaller by a factor of $\frac{\sqrt{3}}{2}$ compared to an $90^{\circ}$ interferometer. However, the sum of three $60^{\circ}$ separated interferometers will enhance the response by a factor of $\sqrt{3}$, giving an overall factor of $\frac{3}{2}$ larger than a single $90^{\circ}$ interferometric detector configuration [38]. The CE's antenna pattern can be written as

$$
\begin{aligned}
F_{+}(\theta, \phi, \psi)= & \frac{1}{2}\left(1+\cos ^{2} \theta\right) \cos 2 \phi \cos 2 \psi \\
& -\cos \theta \sin 2 \phi \sin 2 \psi, \\
F_{\times}(\theta, \phi, \psi)= & -\frac{1}{2}\left(1+\cos ^{2} \theta\right) \cos 2 \phi \sin 2 \psi \\
& -\cos \theta \sin 2 \phi \cos 2 \psi .
\end{aligned}
$$

\section{B. Duration of binary neutron star signals}

In a GW detector, different noise sources limit the sensitivity of the detector in different frequency bands [39]. For example, from 1 to $10 \mathrm{~Hz}$, seismic noise and gravity gradient noise are the main contributors. From 10 to $200 \mathrm{~Hz}$, the performance is limited by quantum noise and thermal noise from the suspension and mirror coatings. For frequencies beyond $200 \mathrm{~Hz}$, shot noise is the primary source of noise [40].
There are many proposed technologies designed to improve the sensitivity of the ET [41-43]. The ET is expected to have improved sensitivity across the frequency band accessible to ground-based detectors compared to second generation detectors. For CE, the exact technologies that will be employed are still undergoing research and study. The current sensitivity curve of CE is obtained using the existing technologies and well defined extrapolations from them. As $\mathrm{CE}$ will make use of the available technologies at the time that it is built, the sensitivity curve should not be considered the design target of CE [37]. The estimated amplitude spectrum density of the ET [43] and $\mathrm{CE}$ are presented in Fig. 1. The design sensitivity of aLIGO and Advanced VIRGO are also shown for comparison. Compared to aLIGO and Advanced Virgo, the ET's sensitivity from $10 \mathrm{~Hz}$ is improved by at least a factor of 10 , the improvement can even be up to a factor of $10^{6}$ in the frequency band below $10 \mathrm{~Hz}$. CE's sensitivity is also improved by up to a factor of $10^{3}$ from 1 to $10 \mathrm{~Hz}$ compared to second generation detectors, and is improved by $\sim 30$ times above $10 \mathrm{~Hz}$.

Different relative improvements in sensitivity as a function of frequency can lead to different impacts on the sky localization capability. Better sensitivity in the medium to high frequency band can effectively increase the SNR for a GW event and thus reduce the localization error. On the other hand, improvement in the low frequency band might not increase the SNR as much, but it will substantially extend the in-band duration of the signal from the order of seconds/minutes to the order of hours/days. We can express the time remaining prior to merger for a compact binary system as a function of the instantaneous $\mathrm{GW}$ frequency [44] using

$$
\tau_{\mathrm{c}}=\frac{5}{256} \frac{c^{5}}{G^{\frac{5}{3}}} \frac{\left(\pi f_{\mathrm{s}}\right)^{-\frac{8}{3}}}{\mathcal{M}^{\frac{5}{3}}}
$$

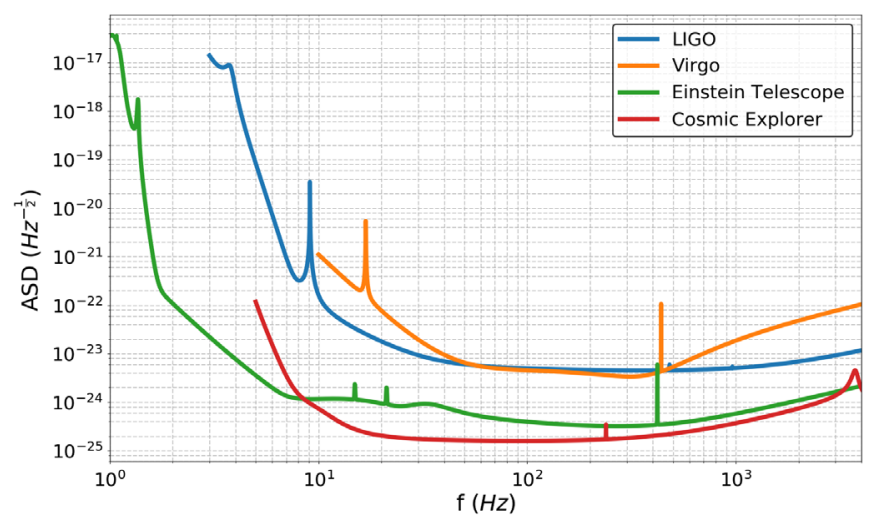

FIG. 1. The amplitude spectrum density for the ET (Green), CE (Red), aLIGO Hanford/Livingston (blue) and Advanced Virgo (Orange). Both aLIGO and Advanced Virgo are at their respective design sensitivities. 


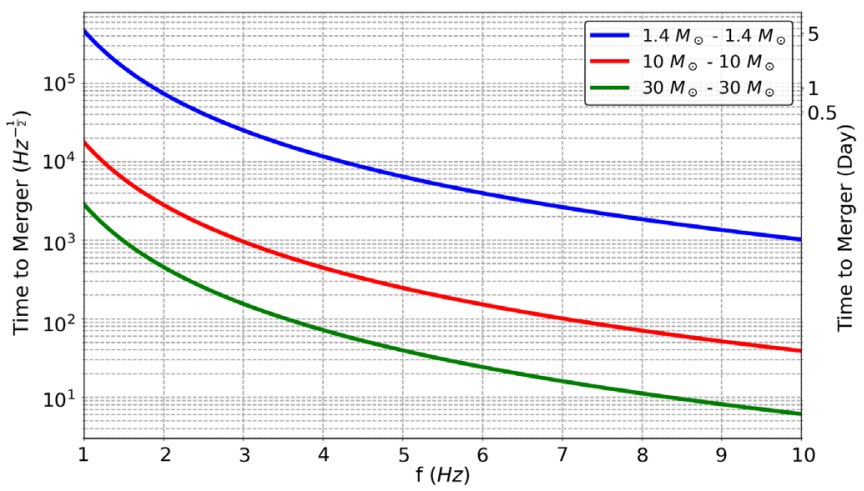

FIG. 2. The time to merger as a function of starting frequency $f_{\mathrm{s}}$ for $1.4 M_{\odot}-1.4 M_{\odot} \mathrm{BNS}$ (blue), $10 M_{\odot}-10 M_{\odot} \mathrm{BBH}$ (red) and $30 M_{\odot}-30 M_{\odot} \mathrm{BBH}$ (green).

where $\tau_{\mathrm{c}}$ is the time to merger, $c$ the speed of light, $G$ the gravitational constant, $\mathcal{M}$ the chirp mass and $f_{\mathrm{s}}$ the starting frequency considered for the GW. Figure 2 shows the time to merger for a $1.4 M_{\odot}-1.4 M_{\odot}$ merger as a function of $f_{\mathrm{s}}$. Also plotted are $10 M_{\odot}-10 M_{\odot}$ and $30 M_{\odot}-$ $30 M_{\odot} \mathrm{BBH}$ mergers for comparison. The in-band duration of a GW from a given compact binary system in a detector can be obtained by replacing the starting frequency $f_{\mathrm{s}}$ with the low frequency cut-off of the detector in Eq. (4). As indicated in Fig. 2, for BNS systems, if the detector's low frequency cut-off is reduced to $2 \mathrm{~Hz}$, the in-band duration of the signal will be close to 1 day, and will be more than 5 days if the low frequency cutoff is $1 \mathrm{~Hz}$. This is substantially longer than the in-band duration for aLIGO and Advanced VIRGO, where the low frequency cutoff is $10 \mathrm{~Hz}$. The in-band duration of BBH signals are expected to be shorter. For the ET with low cutoff frequency at $1 \mathrm{~Hz}$, $10 M_{\odot}-10 M_{\odot} \mathrm{BBH}$ signals will last for $\sim 5$ hours.

Such a long duration allows the detector to observe the signal along the detector's trajectory on earth as the earth rotates, and therefore makes the detector's response explicitly time-dependent. To illustrate this time-dependence, in Fig. 3 two source sky locations are selected and the change over 5 days of the ET and CE detector response to sources at those locations is shown.

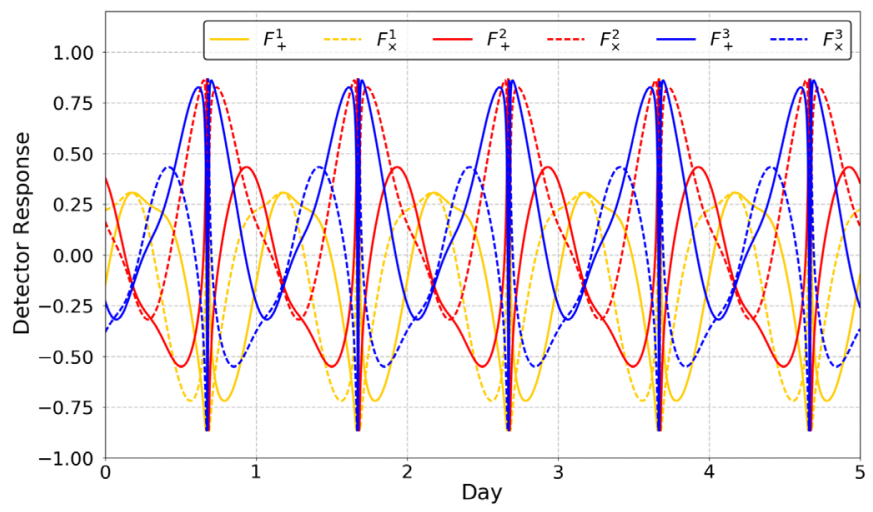

(a)

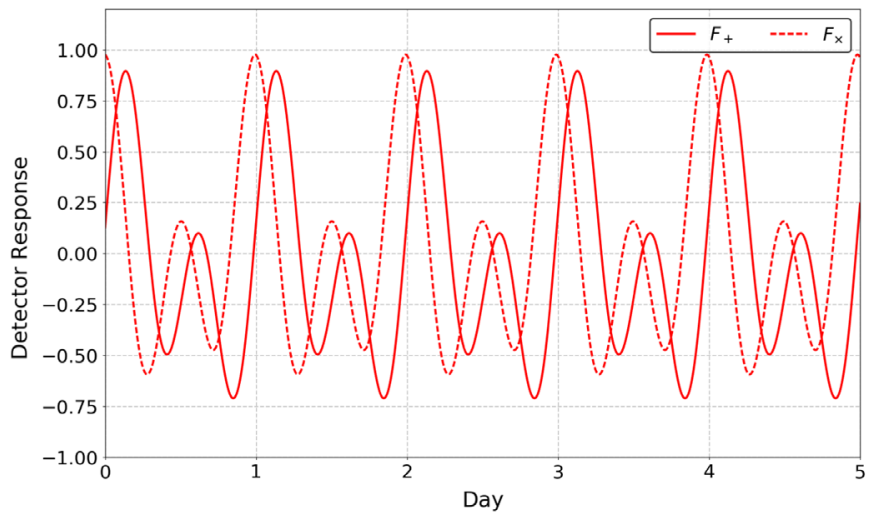

(c)

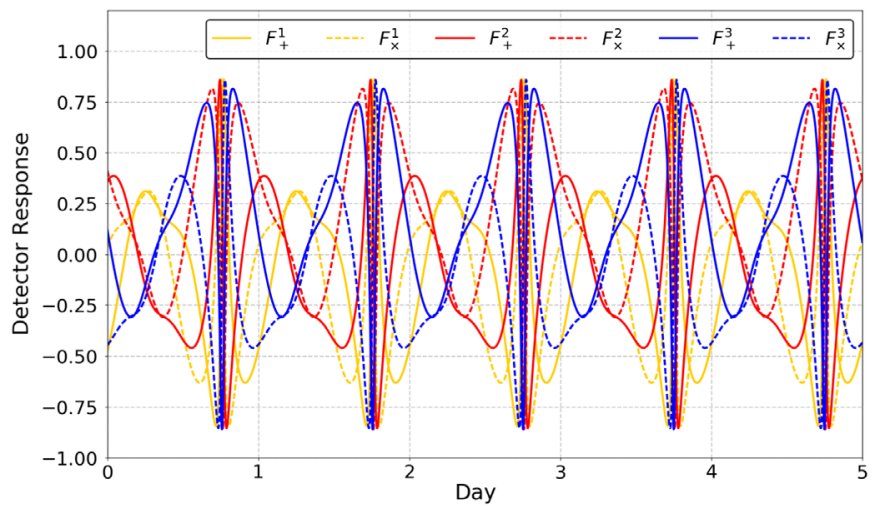

(b)

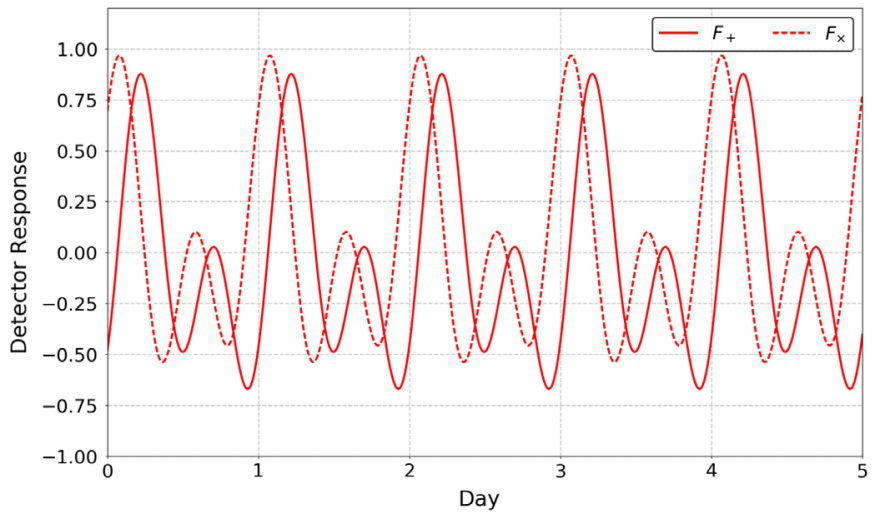

(d)

FIG. 3. The time-dependency of ET's and CE's detector response to GWs with a polarization angle equal to $\pi / 8$ coming from two example locations in the sky over the course of 5 days. Panel a and b are for the ET and panel $\mathrm{c}$ and $\mathrm{d}$ for CE. Panel a and $\mathrm{c}$ show the detector response to a source located at $(\alpha, \delta)=\left(0^{\circ}, 45^{\circ}\right)$ and panel b and d show that to a source at $(\alpha, \delta)=\left(30^{\circ}, 60^{\circ}\right)$, where $\alpha$ and $\delta$ are right ascension and declination of the source. In the legend in panel a and $\mathrm{b}$, the superscript $k=(1,2,3)$ indicates the $k$ th interferometer of the ET. 


\section{METHODOLOGY}

Using the Fisher matrix, we aim to provide a lower bound on source sky position error for GW sources and examine the feasibility of early warning. It is often reported that the Fisher matrix approach produces estimates that are more optimistic than those methods that completely explore the likelihood such as Monte Carlo Markov Chain (MCMC). However, this is often due to the misuse of the Fisher matrix in situations where the SNR is too low. In the moderate to high SNR regime where the Cramer-Rao lower bound is valid [45-47], estimates from the Fisher matrix are a good indicator of the expected uncertainty on parameters. Furthermore, complete MCMC simulations are usually too computationally expensive to carry out, while the Fisher matrix is low to moderate in computational cost.

For an incoming $\mathrm{GW}$, the strain observed by the Ith detector can be expressed as $h_{I}(\boldsymbol{\theta}, t)$ in the time domain. It is a linear combination of the wave's two polarizations $h_{+}(\boldsymbol{\theta}, t), h_{\times}(\boldsymbol{\theta}, t)$ and the detector response $F_{I}^{+}(\theta, \phi, \psi, t)$, $F_{I}^{\times}(\theta, \phi, \psi, t)$ as

$h_{I}(\boldsymbol{\theta}, t)=F_{I}^{+}(\theta, \phi, \psi, t) h_{+}(\boldsymbol{\theta}, t)+F_{I}^{\times}(\theta, \phi, \psi, t) h_{\times}(\boldsymbol{\theta}, t)$,

where the vector $\boldsymbol{\theta}$ represents the unknown signal parameters: sky position, distance, time of arrival at the center of the earth, binary masses, initial phase of the wave when it arrives at the center of the earth, inclination angle and polarization angle. The time at the detector is denoted by $t$ which is equal to the arrival time $t_{0}$ of the incoming wave at the center of the earth, plus the time $\tau$ required for the wave to travel from the center of the earth to the detector, given by

$$
\tau=\frac{\mathbf{n} \cdot \mathbf{r}}{c},
$$

where $\mathbf{n}$ is the GW propagation direction and $\mathbf{r}$ is the location vector of the detector relative to the center of the Earth. The Fourier transform of $h_{I}(\boldsymbol{\theta}, t)$ is then defined as

$$
\tilde{h}_{I}(\boldsymbol{\theta}, f)=\int_{t}^{t+\Delta t} h_{I}(\boldsymbol{\theta}, t) e^{-2 i \pi f t} d t
$$

where $\Delta t$ refers to the duration of $h_{I}(\boldsymbol{\theta}, t)$. The start and the end of $h_{I}(\boldsymbol{\theta}, t)$ are denoted by $t$ and $t+\Delta t$. The mathematical definition of the Fisher matrix is given by

$$
\Gamma_{i j}=\sum_{I=1}^{N} 2 \int_{0}^{\infty} \frac{\frac{\partial \tilde{h}_{l}^{*}(\boldsymbol{\theta}, f)}{\partial \theta_{i}} \frac{\partial \tilde{h}_{I}(\boldsymbol{\theta}, f)}{\partial \theta_{j}}+\frac{\partial \tilde{h}_{l}^{*}(\boldsymbol{\theta}, f)}{\partial \theta_{j}} \frac{\partial \tilde{h}_{I}(\boldsymbol{\theta}, f)}{\partial \theta_{i}}}{S_{I}(f)} d f .
$$

where $\partial \tilde{h}(\boldsymbol{\theta}, f) / \partial \theta_{i}$ is the partial derivative of $\tilde{h}(\boldsymbol{\theta}, f)$ with respect to the $i$ th unknown parameter $\theta_{i}$. The power spectrum density of the $I^{\text {th }}$ detector is denoted by $S_{I}(f)$. We also sum over the number of detectors, or in the case of the ET, over the number of individual interferometers. The optimal SNR, $\rho$, of the incoming GW can be expressed as

$$
\rho^{2}=4 \int_{0}^{\infty} \frac{\left|\tilde{h}_{I}(\boldsymbol{\theta}, f)\right|^{2}}{S_{I}(f)} d f .
$$

In this work, we construct the Fisher matrix for the following unknown parameters: right ascension, $\alpha$; declination, $\delta$; arrival time, $t_{0}$, at the center of the earth; the log of the distance, $\log _{10} d$; polarization angle, $\psi$; the log of the total binary masses, $\log _{10} M$; the cosine of the inclination angle, $\cos l$; the symmetric mass ratio, $\eta=M_{1} \times M_{2} / M^{2}$, of the masses of the two bodies in the binary; the initial phase, $\phi_{0}$, of the wave when it arrives at the center of the earth. Specifically, the equatorial coordinates $\alpha$ and $\delta$ of a source are related to $\theta$ and $\phi$ in Eqs. (1)-(3) by rotation matrices once detector location and the time of observation are specified.

When computing the $\mathrm{GW}$ localization error for a source at a particular sky location, we divide the entire wave into pieces, each of which is 100 seconds long — with the final piece $\leq 100$ seconds depending on the specific in-band duration of the signal. The total number of pieces $N_{\mathrm{p}}$ is then equal to $\tau_{\mathrm{c}} / 100$, rounded towards positive infinity [i.e., $\Delta t \leq 100 \mathrm{~s}$ in Eq. (7)]. For each piece of the wave, we employ the formalism described above to compute the Fisher matrix $\Gamma_{i j}^{k}$ and the optimal SNR $\rho^{k}$. The superscript $k$ indicates the $k$ th piece of the wave. The final Fisher matrix $\Gamma_{i j}^{\mathrm{f}}$ is then

$$
\Gamma_{i j}^{\mathrm{f}}=\sum_{k=1}^{N_{p}} \Gamma_{i j}^{k},
$$

where we sum over the Fisher matrix contributions from each piece of the waveform, and the superscript $f$ indicates the resultant Fisher matrix. The matrix inverse of the Fisher matrix then gives the covariance matrix of the unknown parameters as

$$
\operatorname{cov}_{i j}=\Gamma_{i j}^{-1},
$$

from which the localization error is extracted using

$$
\Delta \Omega=2 \pi \sqrt{\lambda_{\alpha} \lambda_{\delta}} \cos \delta
$$

where $\Delta \Omega$ is the localization uncertainty, $\lambda_{\alpha}$ and $\lambda_{\delta}$ are the eigenvalues of the matrix $\operatorname{cov}_{i j}$ corresponding to the $\alpha$ and $\delta$ of the source respectively. The following expression can be used to convert $\Delta \Omega$ to any desired confidence level,

$$
\Delta \Omega_{p}=-2 \log (1-p) \Delta \Omega,
$$

where $p$ is a value between 0 and 1 indicating the confidence level. Similarly, the accumulated SNR is given by

$$
\left(\rho^{f}\right)^{2}=\sum_{k=1}^{N_{p}}\left(\rho^{k}\right)^{2} .
$$




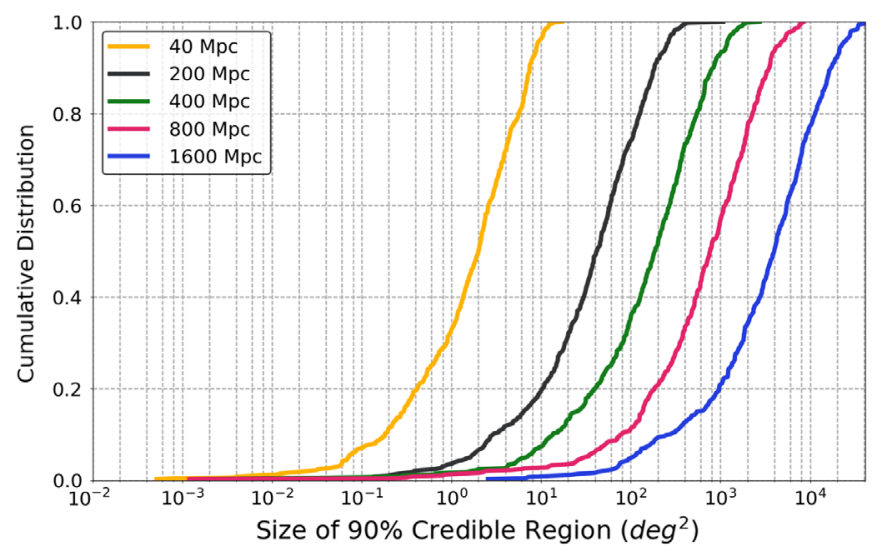

(a)

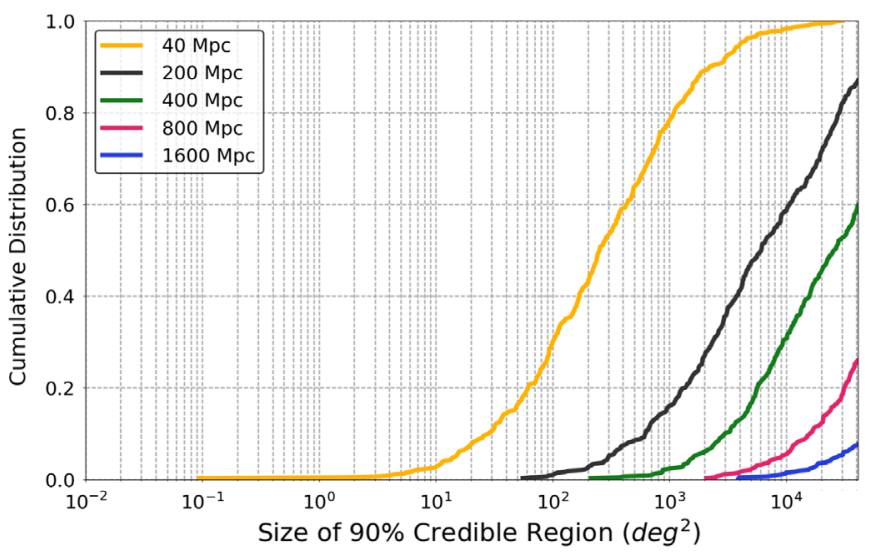

(b)

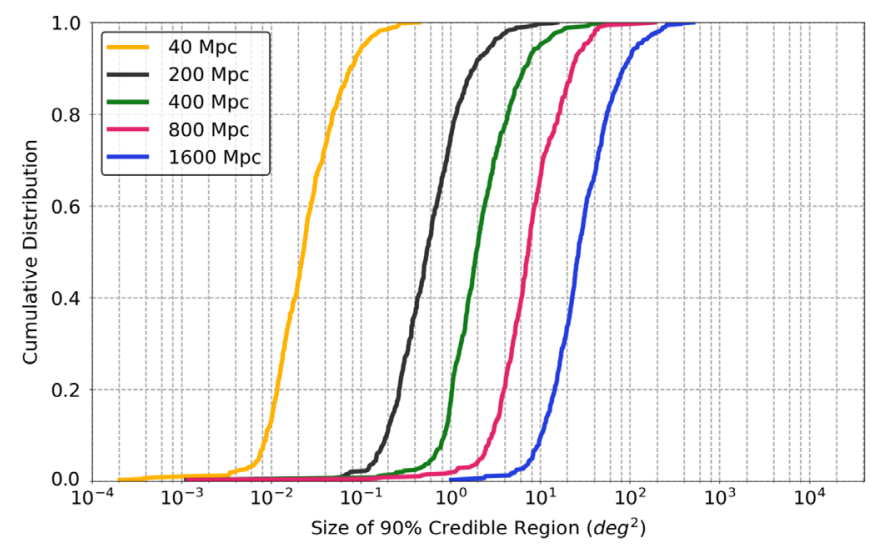

(c)

FIG. 4. The cumulative distribution of the size of $90 \%$ credible regions for sources at fixed distances. The x-axes show the size of the $90 \%$ credible region and the upper limit of the $\mathrm{x}$-axes corresponds to the size of the whole sky. The yellow, black, green, red, and blue lines represent BNS sources at 40, 200, 400, 800, and $1600 \mathrm{Mpc}$ respectively. Panel a and b show the results for the ET and CE respectively. Panel c shows the results for the ET and CE as a network.

\section{RESULTS OF SIMULATION AND DISCUSSION}

\section{A. Localization}

To test the localization capabilities of third generation detectors, we have simulated GW signals from $1.4 M_{\odot}-$ $1.4 M_{\odot}$ BNS sources at distances of 40, 200, 400, 800, and $1600 \mathrm{Mpc}$. The masses are defined in the local frame, i.e., $M_{\text {Local }}$, which is related to the observed masses $M_{\mathrm{Obs}}$ by

$$
M_{\text {Obs }}=M_{\text {Local }}(1+z)
$$

We use $z$ to denote redshift. All the masses defined earlier refer to the observed masses $M_{\mathrm{Obs}}$. The inclination angle $l$, polarization angle $\psi$ and the sky position $(\alpha, \delta)$ are randomized. For each specific distance, we have simulated 500 BNS signals. To determine whether a source is detectable, we have employed different SNR cuts for each network configuration. For networks with more than one interferometer such as the ET or the ET and CE, we have applied an SNR requirement similar to that in [27]. A detection is achieved if the network SNR is larger than or equal to 12 and the SNRs in at least two interferometers are no less than 5.5. For a network with only one interferometer, namely, $\mathrm{CE}$, we require that the accumulated SNR is no less than 12 to claim a detection. ${ }^{1}$ The results of the simulations are presented as cumulative distributions in Fig. 4.

For BNSs at $40 \mathrm{Mpc}$ using only the ET, $50 \%$ of the detectable sources can be localized with $90 \%$ confidence to within $2.0 \mathrm{deg}^{2}$, and $90 \%$ of the detectable sources to within $7.5 \mathrm{deg}^{2}$. For the best localized $10 \%$ of sources, the $90 \%$ credible region is within $0.2 \mathrm{deg}^{2}$ and these correspond to the best located and orientated sources. For BNSs at $200 \mathrm{Mpc}, 50 \%$ and $90 \%$ of the detectable sources can be localized with $90 \%$ confidence to within

\footnotetext{
${ }^{1}$ We require a single detector to achieve $\mathrm{SNR} \geq 12$ for a detection for consistency. The results shown here for a single detector may therefore be more pessimistic than the reality.
} 
$42 \mathrm{deg}^{2}$ and $183 \mathrm{deg}^{2}$ respectively. Assuming EM followup observations are achievable for sources that are localized to within $100 \mathrm{deg}^{2}$, this indicates $100 \%$ (74\%) of the detectable sources at $40 \mathrm{Mpc}(200 \mathrm{Mpc})$, suggesting many opportunities for joint EM observations provided by the ET for BNSs within $200 \mathrm{Mpc}$.

For sources located at $400 \mathrm{Mpc}$, the upper limit of the size of $90 \%$ credible region increases to $187 \operatorname{deg}^{2}\left(812 \mathrm{deg}^{2}\right)$ for the best localized 50\% (90\%) of the detectable sources. This still leaves $36 \%$ of the detectable sources localized to within $100 \mathrm{deg}^{2}$ with 90\% confidence. For sources located at larger distances, i.e., 800 and $1600 \mathrm{Mpc}$, the upper limit of the size of $90 \%$ credible regions for the best localized $50 \%$ (90\%) of the detectable sources increases substantially to $764 \mathrm{deg}^{2}$ $\left(3485 \mathrm{deg}^{2}\right)$ and $3994 \mathrm{deg}^{2}\left(1.7 \times 10^{4} \mathrm{deg}^{2}\right)$ respectively. Moreover, only $11 \%$ and $5 \%$ of the detectable sources can be localized to within $100 \mathrm{deg}^{2}$. This is because the amplitude of the signals from sources at greater distances will be weaker. Also, the observed $M_{\mathrm{Obs}}$ as defined in Eq. (15) will be larger, meaning that the in-band duration will be shorter. This suggests that localization of a BNS at such distances by the ET alone will still be poor and EM follow-up observations will remain a challenge if the ET is the only operating detector.

Since the sensitivity of CE at low frequencies is limited, the in-band durations of the signals are shorter than that in the ET. As shall be seen later, the time-dependent modulation of detector response is the main factor contributing to improved localization. Consequently, the localization of a BNS by CE alone is worse. For example, $50 \%$ (90\%) of the detectable BNSs at $40 \mathrm{Mpc}$ can be localized to only within $252 \operatorname{deg}^{2}\left(2212 \mathrm{deg}^{2}\right.$ ), a factor of $\sim 126$ and 295 larger than using only the ET. Only $30 \%$ of the detectable sources can be localized to within $100 \mathrm{deg}^{2}$ with $90 \%$ confidence. For sources at distances $\geq 400 \mathrm{Mpc}$, the upper limits of localization error for the best localized $50 \%$ and $90 \%$ are larger than the whole sky. This means that for some sources, despite accumulating enough SNR to claim a detection, no localization information is available.

Combining the ET and CE together as a network greatly improves the localization since it vastly increases the geographical baseline of the network. This greatly improves triangulation between the detectors in the network and will take advantage of the high frequency, high SNR components of the waveform, i.e., the final seconds. This will complement the localization information gained from the long duration and changing antenna patterns. All sources within $200 \mathrm{Mpc}$ are localized to within $30 \mathrm{deg}^{2}$ with $90 \%$ confidence. Importantly, at 40 and $200 \mathrm{Mpc}$, the 90\% credible region upper limit for the best localized $90 \%$ of the detectable sources are only $\mathcal{O}\left(10^{-2}\right) \mathrm{deg}^{2}$ and $\mathcal{O}(1) \mathrm{deg}^{2}$ respectively. For the detectable sources at $1600 \mathrm{Mpc}$, there are still $92 \%$ localized to within $100 \mathrm{deg}^{2}$ with $90 \%$ confidence. This shows a great promise

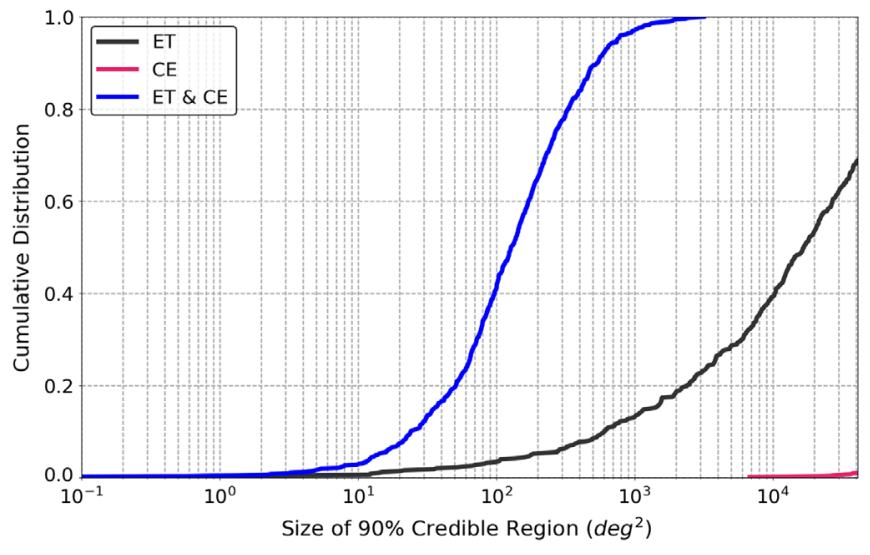

FIG. 5. The cumulative distribution of the size of $90 \%$ credible regions in the sky, for detectable BNS sources uniformly distributed in comoving volume, observed by the ET and CE both individually and as a network. The upper limit of the $\mathrm{x}$-axis corresponds to the size of the entire sky.

for BNS multimessenger astronomy even at relatively large cosmological distances.

To present a more general picture, we have also simulated the localization of a population of BNS that are distributed uniformly in the comoving volume. The results are presented in Fig. 5 as cumulative distributions. Using the ET alone, the farthest detectable source is at $z=1.7$. Of the detectable sources, $50 \%$ can be localized to within $\sim 1.7 \times 10^{4} \mathrm{deg}^{2}$ with $90 \%$ confidence. The cumulative distribution for the ET reaches $68 \%$ when the value at the $\mathrm{x}$-axis is the size of the entire sky-i.e., this indicates that for up to $32 \%$ of the detectable sources, essentially no localization information is available. For CE, the situation is worse. The farthest detectable source is located at $z=4.9$, but only $\sim 2 \%$ of the detectable sources will have any localization information available. Again, a network with the ET and CE can bring a huge improvement to the localization performance. For example, compared to using the ET only, the upper limit of the $90 \%$ credible region for the best localized $90 \%$ of the detectable sources has been reduced by a factor $>100$ to $123 \mathrm{deg}^{2}$. The fraction of detectable sources that can be localized to within $100 \mathrm{deg}^{2}$ with $90 \%$ confidence has increased by more than 10 times to $43 \%$. Interestingly, the farthest detectable source with a network of the ET and CE is located at $z=2.2$. This is because for a network of more than one interferometer, we require an SNR $\geq 5.5$ in at least two of the interferometers, besides also requiring a network $S N R \geq 12$. For sources located at $z>2.2$, only the $\mathrm{CE}$ is able to accumulate enough SNR-leading to a failure to meet the detection criterion.

A summary of the results is given in Table I. Given the success of the EM follow-up observations of GW170817, where the localization error at $90 \%$ confidence is $28 \mathrm{deg}^{2}$ [6], also presented in the table is a column showing the 
TABLE I. Statistical summary of results.

\begin{tabular}{|c|c|c|c|c|c|c|}
\hline Network & $\mathrm{d}(\mathrm{Mpc})$ & $n$ & $50 \%\left(\mathrm{deg}^{2}\right)$ & $90 \%\left(\operatorname{deg}^{2}\right)$ & $\leq 100\left(\operatorname{deg}^{2}\right)$ & $\leq 30\left(\operatorname{deg}^{2}\right)$ \\
\hline \multirow[t]{6}{*}{ ET } & 40 & & 2 & 8 & $100 \%$ & $100 \%$ \\
\hline & 200 & & 42 & 183 & $74 \%$ & $40 \%$ \\
\hline & 400 & 500 & 187 & 837 & $36 \%$ & $16 \%$ \\
\hline & 800 & & 764 & 3485 & $11 \%$ & $5 \%$ \\
\hline & 1600 & & 3994 & $1.7 \times 10^{4}$ & $5 \%$ & $2 \%$ \\
\hline & Uniform $^{\mathrm{a}}$ & 3000 & $1.7 \times 10^{4}$ & $>$ Sky & $3 \%$ & $2 \%$ \\
\hline \multirow[t]{6}{*}{$\mathrm{CE}$} & 40 & & 252 & 2212 & $30 \%$ & $10 \%$ \\
\hline & 200 & & 6118 & $>$ Sky & $1 \%$ & $0 \%$ \\
\hline & 400 & 500 & $2.6 \times 10^{4}$ & $>$ Sky & $0 \%$ & $0 \%$ \\
\hline & 800 & & $>$ Sky & $>$ Sky & $0 \%$ & $0 \%$ \\
\hline & 1600 & & $>$ Sky & $>$ Sky & $0 \%$ & $0 \%$ \\
\hline & Uniform $^{\mathrm{a}}$ & 5000 & $>$ Sky & $>$ Sky & $0 \%$ & $0 \%$ \\
\hline \multirow[t]{6}{*}{ ET \& CE } & 40 & & $2 \times 10^{-2}$ & $8 \times 10^{-2}$ & $100 \%$ & $100 \%$ \\
\hline & 200 & & $5 \times 10^{-1}$ & 1.8 & $100 \%$ & $100 \%$ \\
\hline & 400 & 500 & 2 & 7 & $100 \%$ & $99 \%$ \\
\hline & 800 & & 7 & 23 & $99 \%$ & $94 \%$ \\
\hline & 1600 & & 27 & 85 & $92 \%$ & $55 \%$ \\
\hline & Uniform $^{\mathrm{a}}$ & 5000 & 128 & 538 & $41 \%$ & $12 \%$ \\
\hline
\end{tabular}

\begin{abstract}
${ }^{\mathrm{a}}$ Uniformly distributed in the comoving volume.
A brief statistical summary of our results for sky localization. In the first row, we use $d$ to denote distance and $n$ the number of injections. The third and the fourth columns indicate the upper limit of the size of $90 \%$ credible regions for the best localized $50 \%$ and $90 \%$ of the detectable sources. The fifth column shows the percentage of the detectable sources that can be localized to within $100 \mathrm{deg}^{2}$ with $90 \%$ confidence, and the last column the percentage within $30 \mathrm{deg}^{2}$ with $90 \%$ confidence.
\end{abstract}

percentage of detectable sources that can be localized to within $30 \mathrm{deg}^{2}$ with $90 \%$ confidence.

\section{B. Early warning}

In the era of third generation detectors, due to the extended in-band duration of detectable signals, it is possible that signals will accumulate SNR such that the trigger may be considered significant before the merger occurs. In this section we investigate the feasibility of issuing early warnings prior to binary coalescence. We assume that if the SNR for a GW event can be accumulated before merger, up to a level that satisfies the detection requirement as defined in Sec. IVA, the event will be deemed significant. As the purpose of releasing an early warning is to increase the chance of successful EM followup observation, releasing an alert too early may result in a localization error too large to carry out any meaningful follow-ups. We therefore require two criteria to be met before an alert can be released. First, the signal has to satisfy the SNR requirement for detection and secondly, the $90 \%$ credible region has to be no larger than $100 \mathrm{deg}^{2}$ at the moment the alert is sent. The latter requirement is chosen to be consistent with the sky coverage of the EM follow-up campaign for the first detected GW event GW150914 by optical telescopes with $\lesssim 10 \mathrm{deg}^{2}$ fields of view [48]. This is also consistent with the number of fields these telescopes are able to observe in an hours-long observation targeting kilonovae associated with BNS mergers [49]. We will refer to these two requirements as early warning criteria in the remaining of this paper. As early warning is mostly made possible due to the improvement in the sensitivity in the low frequency band, we focus our analysis on the ET, and the ET and CE as a network. The BNS systems are distributed at specific distances and uniformly in the comoving volume as discussed before.

We present the results for the ET in Fig. 6 and the ET and $\mathrm{CE}$ operating together as a network in Fig. 7. These histograms show the distribution of the fraction of detectable events as a function of the time before merger at which the events meet the early warning criteria. Using the ET, all the signals at $40 \mathrm{Mpc}$ meet the early warning criteria between 1 and 20 hours before merger, with the mode of the distribution at $~ 5$ hours. At $200 \mathrm{Mpc}, 58 \%$ of the detectable signals have accumulated enough SNR for early warning between 1 to 6 hours prior to merger. This represents a significant advantage that can be provided by the ET in EM followup observations for sources within $200 \mathrm{Mpc}$. As the distance increases, the fraction of detectable sources that meet the early warning criteria continues to drop. Of the detectable sources at $400 \mathrm{Mpc}$, only 27\% can meet the early warning criteria and the fraction further drops to $\sim 9 \%$ and $\sim 3 \%$ for sources at 800 and $1600 \mathrm{Mpc}$ respectively. Moreover, at $1600 \mathrm{Mpc}$, the times prior to merger when the signals meet the early warning criteria drop to $\leq 50$ minutes. 


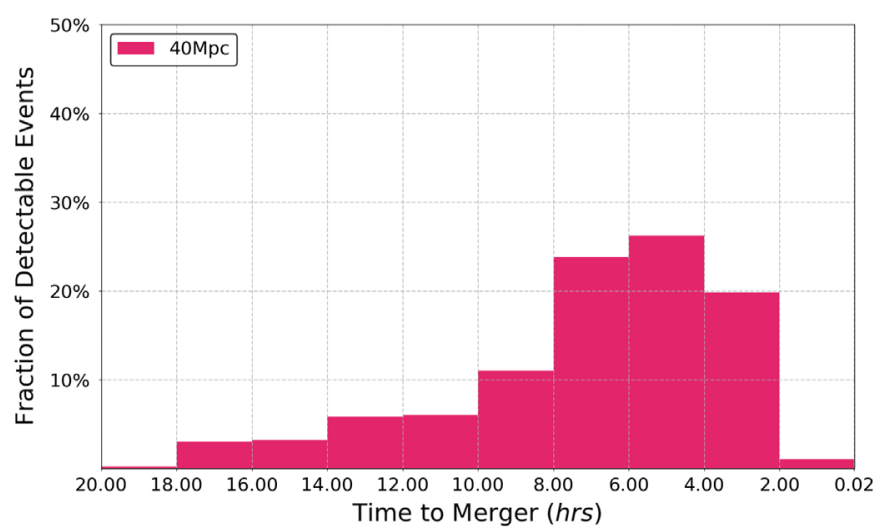

(a)

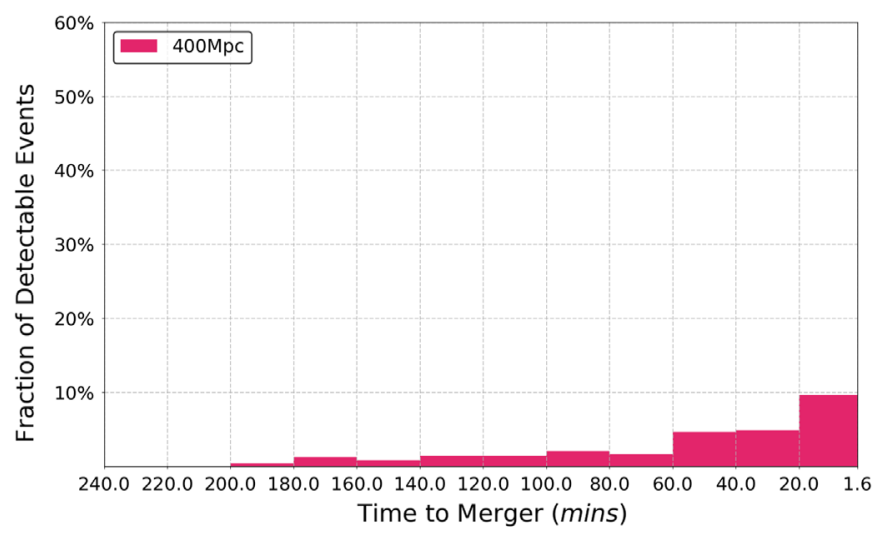

(c)

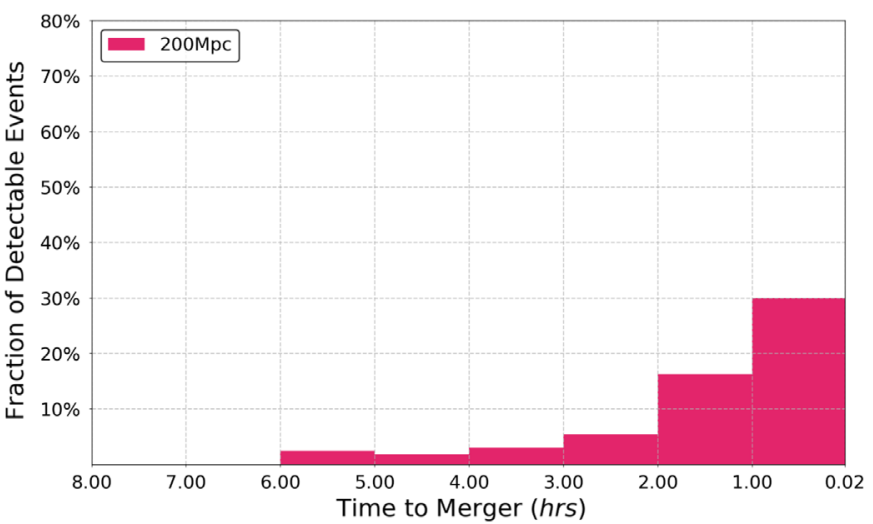

(b)

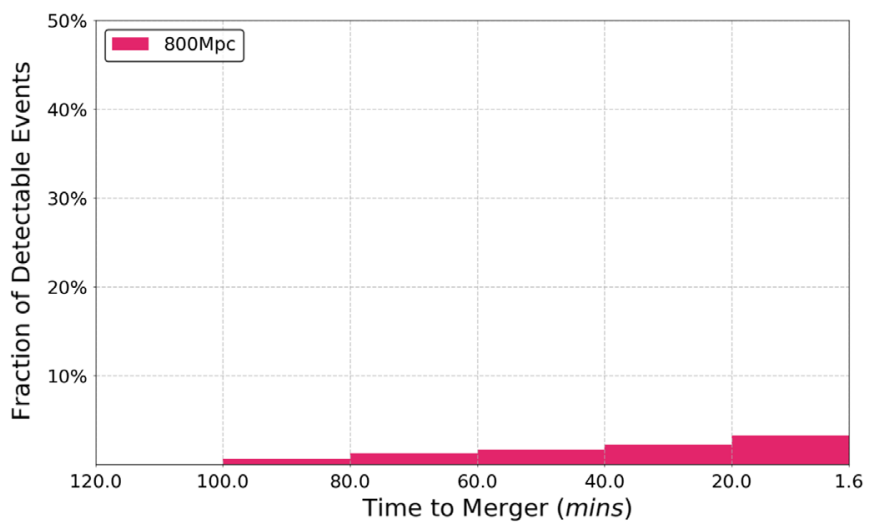

(d)

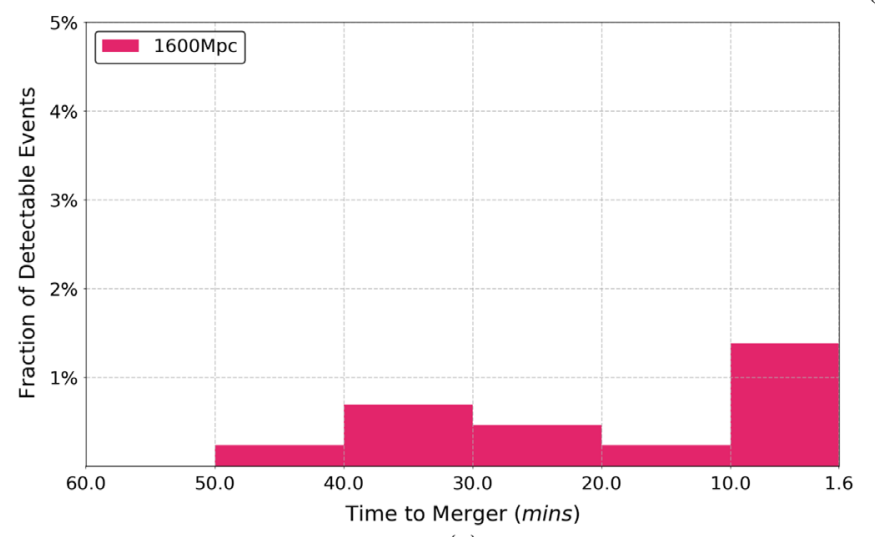

(e)

FIG. 6. Histograms of the fraction of detectable events that achieve the early warning criteria as a function of time to merger for the ET detector. Panel a, b, c, d, and e are for events at 40, 200, 400, 800, and $1600 \mathrm{Mpc}$ respectively. The X-axes indicate the time to merger when the signal meets the early warning criteria. The y-axes indicate the fraction of detectable events that achieve these early warning criteria. Note that at distances $\geq 400 \mathrm{Mpc}$, since a large fraction of the times until merger will fall within 1 hour, for greater clarity the scale of the axes varies from panel to panel. Only those signals which achieve the early warning criteria at least 100 seconds prior to merger will be counted.

As would be expected, an additional third generation detector will improve the performance significantly and provide much improved early warning capability. In Fig. 7, it can be seen that for distances $\geq 200 \mathrm{Mpc}$ and $\leq 1600 \mathrm{Mpc}$, the distributions of early warning times have become noticeably skewed to larger times compared to using only the ET. This suggests that a network of the ET and $\mathrm{CE}$ detectors will provide better early warning capability for sources at relatively large distance. For example, the fractions of the detectable sources at 400, 800, and $1600 \mathrm{Mpc}$ that can meet the early warning criteria are $98 \%, 51 \%$, and 5\% respectively. At $40 \mathrm{Mpc}$, since using only the ET all the sources will have already met the early warning criteria at a time when the frequency of the signal 


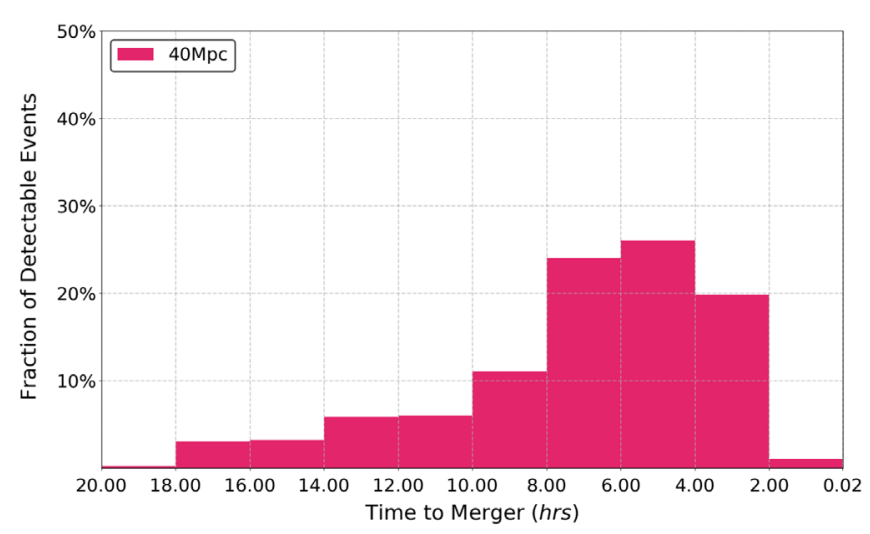

(a)

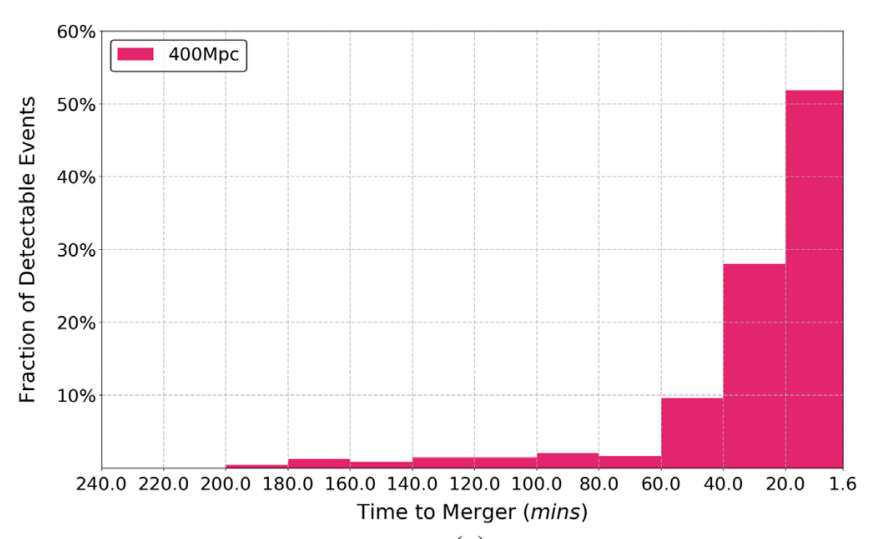

(c)

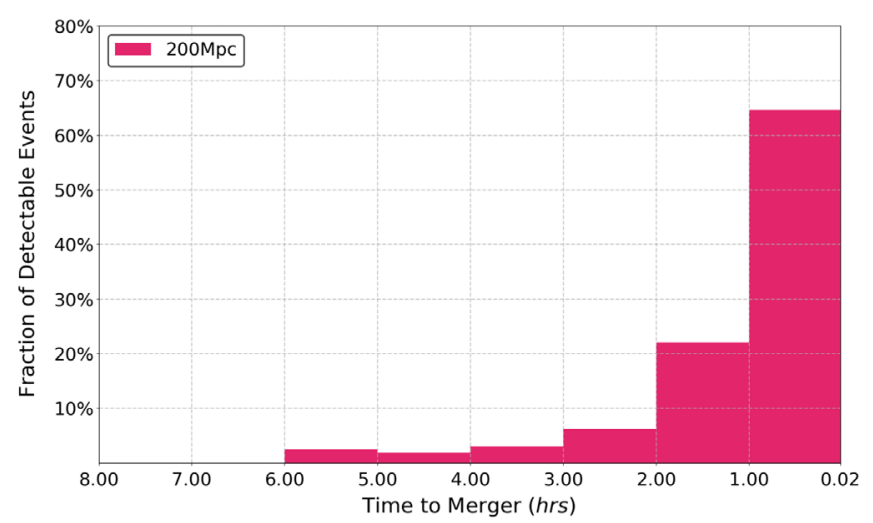

(b)

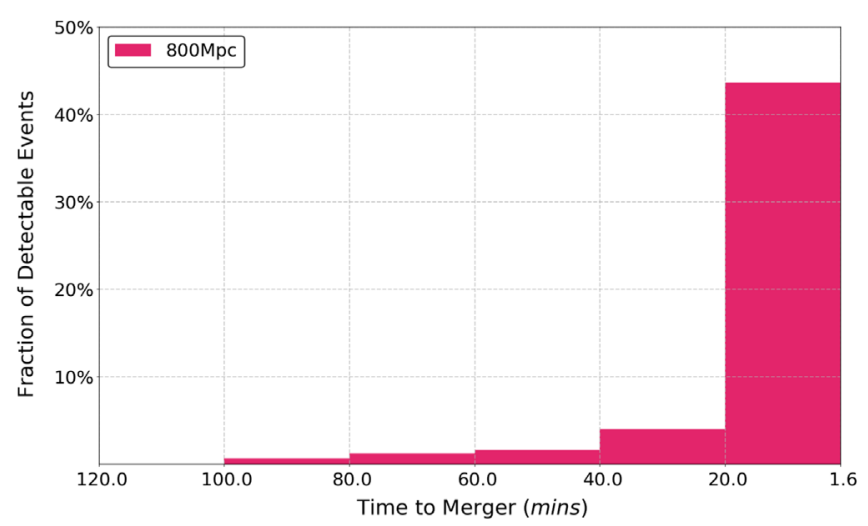

(d)

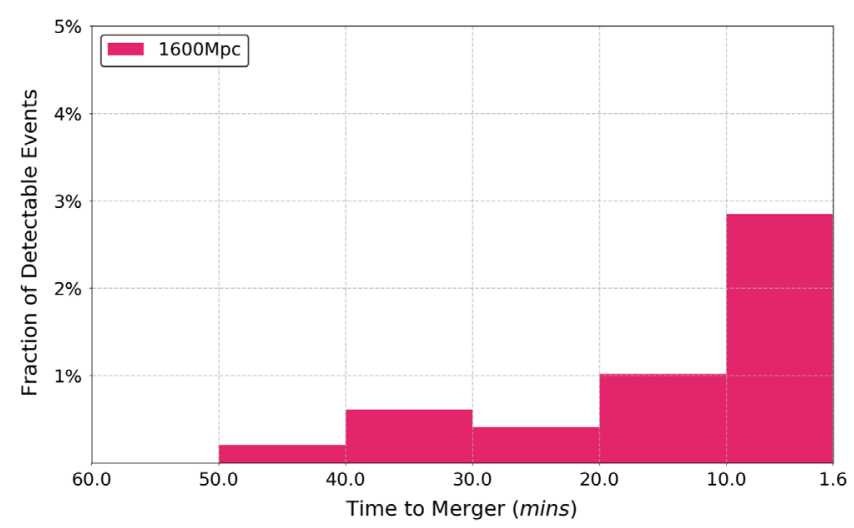

(e)

FIG. 7. The same as Fig. 6 but using the ET and CE as a network. For the same source distance, the scale of the panels is the same as is shown in Fig. 6, to allow a convenient comparison.

is still relatively low, an additional detector of $\mathrm{CE}$ does not alter the distribution significantly. At $1600 \mathrm{Mpc}$, the result may seem to suggest that a network of the ET and CE does not perform much better than using the ET alone. However, this is because a network of the ET and CE will be able to detect sources that are undetectable to the ET alone. These sources will not contribute much to the number of events that meet the early warning criteria but will contribute to the number of detectable events.
Finally, to provide a more general picture, we present in Fig. 8 the results for a population of BNS distributed uniformly in comoving volume. In line with the results shown in the previous sections, a network of the ET and CE will increase the number of events that meet the early warning criteria. With the ET alone, $2 \%$ of detectable sources can have their alerts released prior to merger. This ratio is $\sim 4 \%$ after CE joining the observation. However, the reason for the small increase in the fraction is because a 


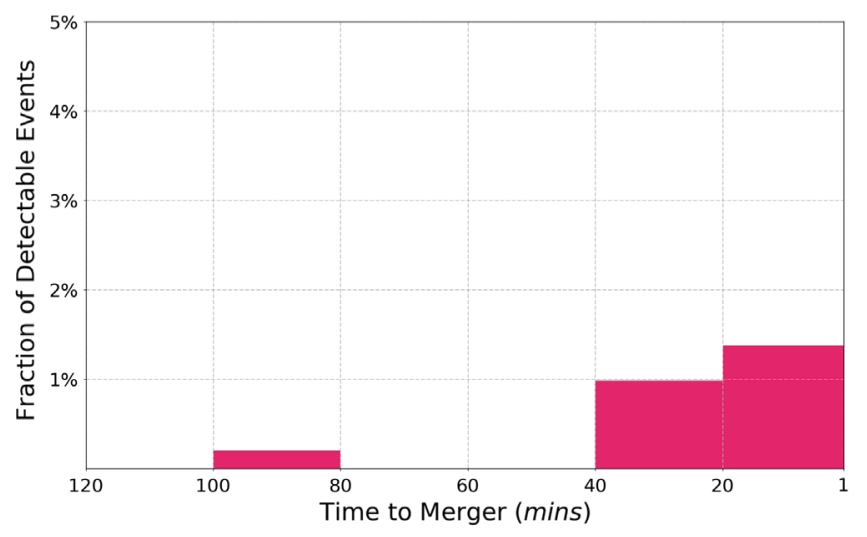

(a)

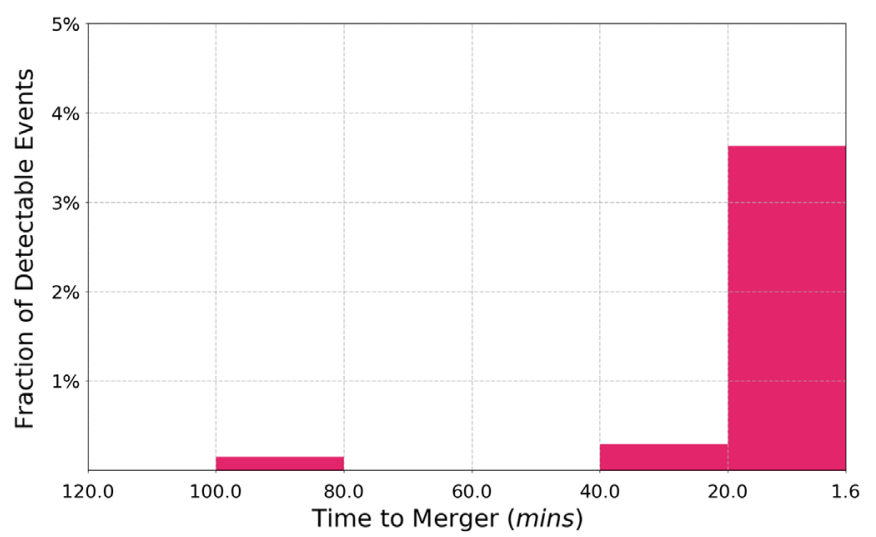

(b)

FIG. 8. Histograms showing the fraction of detectable events that meet the early warning criteria as a function of time to merger for a population of BNS sources distributed uniformly in comoving volume. Panel a shows the results for the ET and panel $\mathrm{b}$ for the ET and CE as a network.

network of the ET and CE will be able to detect sources that are undetectable to the ET alone, and sources located at greater distances. In Table II, we present a summary of the results in terms of early warning.

As discussed, modulations of the Doppler effect and time-dependent detector responses are the two main consequences that will be seen in long in-band duration signals. Zhao and Wen, 2017 [35] has tested thoroughly the difference in localizations with or without including the time-dependencies of these two effects, for networks of third generation detectors. However, it is still not clear which of these two factors has a more important role in terms of localizing BNS mergers. We here investigate the relative importance of these two factors.

To test this, we repeat the simulations for the ET shown in Sec. IVA. While we still enable a time-dependent detector response, we fix the time delay between the center of the earth and the ET at the beginning of the signals. This is because turning on and off the Doppler shift should allow us to see more easily its importance. The results are shown in Fig. 9. It can be seen that at all distances, the cumulative
TABLE II. Statistical summary of results for early warning.

\begin{tabular}{lcrrrrrr}
\hline \hline Network & $\mathrm{d}(\mathrm{Mpc})$ & $n$ & $100 \mathrm{sec}$ & $0.5 \mathrm{hrs}$ & $2 \mathrm{hrs}$ & $5 \mathrm{hrs}$ & $10 \mathrm{hrs}$ \\
\hline ET & 40 & & $100 \%$ & $100 \%$ & $99 \%$ & $66 \%$ & $18 \%$ \\
& 200 & & $58 \%$ & $39 \%$ & $13 \%$ & $2 \%$ & $0 \%$ \\
& 400 & 500 & $28 \%$ & $16 \%$ & $4 \%$ & $0 \%$ & $0 \%$ \\
& 800 & & $9 \%$ & $4 \%$ & $0 \%$ & $0 \%$ & $0 \%$ \\
& 1600 & & $3 \%$ & $1 \%$ & $0 \%$ & $0 \%$ & $0 \%$ \\
& Uniform $^{\mathrm{a}}$ & 3000 & $2 \%$ & $1 \%$ & $0 \%$ & $0 \%$ & $0 \%$ \\
ET \& CE & 40 & & $100 \%$ & $100 \%$ & $99 \%$ & $66 \%$ & $18 \%$ \\
& 200 & & $100 \%$ & $74 \%$ & $13.4 \%$ & $2 \%$ & $0 \%$ \\
& 400 & 500 & $98 \%$ & $27 \%$ & $4 \%$ & $0 \%$ & $0 \%$ \\
& 800 & & $51 \%$ & $4 \%$ & $0 \%$ & $0 \%$ & $0 \%$ \\
& 1600 & & $5 \%$ & $1 \%$ & $0 \%$ & $0 \%$ & $0 \%$ \\
& Uniform $^{\mathrm{a}}$ & 5000 & $4 \%$ & $1 \%$ & $0 \%$ & $0 \%$ & $0 \%$ \\
\hline \hline
\end{tabular}

${ }^{a}$ Uniformly distributed in the comoving volume.

A brief statistical summary of the results for early warning. In the first row, we again use $d$ to denote distance and $n$ the number of injections. The third to the seventh columns indicate the fraction of detectable events that meet the early warning criteria within the corresponding times.

distributions are almost identical, with only marginal discrepancy. This suggests that the Doppler effect is not important and the modulation of the detector response is the main cause of improved sky localization.

\section{Calibration errors}

Previous studies have dealt with calibration errors in the context of second generation detectors [50-53]. We present here a brief discussion of the impact of calibration errors on localization for third generation detectors. It is recognized

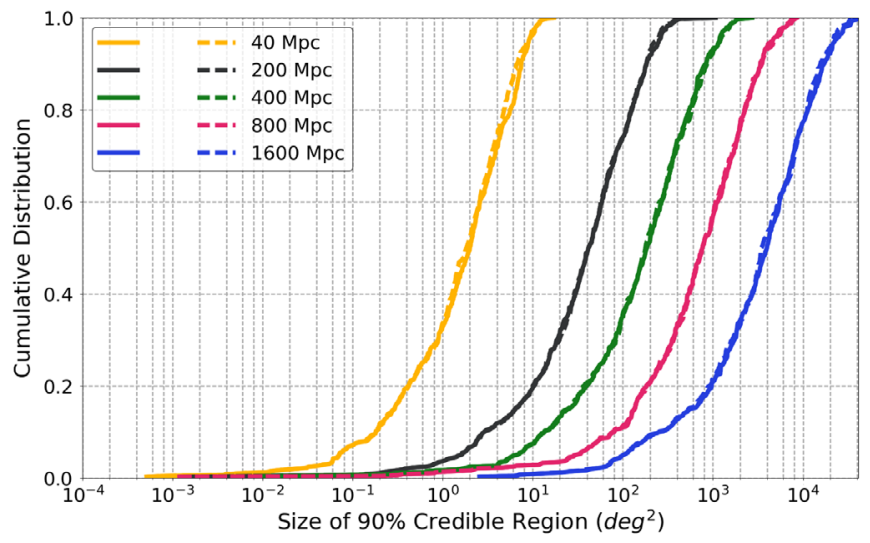

FIG. 9. The cumulative distribution of the size of $90 \%$ credible regions for sources at fixed distances, with and without the Doppler shift effect. The $\mathrm{x}$-axis shows the size of the $90 \%$ credible region and the upper limit of the $\mathrm{x}$-axis corresponds to the size of the whole sky. The yellow, black, green, red, and blue lines represent BNS sources at 40, 200, 400, 800, and $1600 \mathrm{Mpc}$ respectively. The solid lines and the dashed lines show the results with and without including the Doppler shift of the waves respectively. 
that bias in the output of a detector can be introduced by errors in its calibration-i.e., differences between the actual response function and the measured response function of the detector. These differences can then affect the noise and cause amplitude errors and timing errors in the gravitational wave strain used for analysis. Inference on the location of the source of a GW from the output strain can therefore be biased.

Amplitude errors will affect the localization by introducing a bias in the measurement of distance, inclination and polarization angles. With second generation detectors, these parameters cannot be measured precisely. For example, the uncertainty on distance can be $\sim 40 \%$ for an event with SNR 8 [51]. Therefore, systematic errors caused by amplitude uncertainties are not expected to be dominant. However, the fractional uncertainty on distance of a GW from BNS is inversely proportional to SNR. It is conceivable that when the ET and CE are operational, higher SNR and the extended in-band duration will increase the accuracy with which these parameters can be determined. The amplitude error-induced bias may therefore be comparable to the uncertainty on the measurement of the parameters. Moreover, we assumed in this work that the actual value of the detector response will agree with the theoretical calculation. As shown in Fig. 9, the time evolution of the detector response is crucial for localization of BNS mergers with third generation detectors. Any uncertainty in the amplitude of the waves or the detector response will certainly affect that. As a result, the inference without accounting for these errors may systematically shift the probable locations of the source away from its true location. Amplitude errors are therefore expected to have a larger effect in parameter estimation for third generation detectors and need to be quantified.

Localization can also be affected by timing errors of a signal through timing triangulation. The accuracy with which the arrival time of a signal is determined is inversely proportional to the SNR of a wave cycle at the frequencies at which the detectors are most sensitive. For advanced detectors, such as aLIGO and Advanced VIRGO, this happens at $\sim 100 \mathrm{~Hz}$ giving a timing accuracy $\mathcal{O}\left(10^{-3}\right)$ seconds. Timing errors (i.e., the errors intrinsic to timing when the data sample is taken) therefore would have to be comparable to a millisecond in order to be significant. However, as third generation detectors will have improved sensitivity, the SNRs for a fraction of detectable sources will therefore be high enough that timing error may be significant. It is therefore necessary to quantify timing errors for third generation detectors.

\section{CONCLUSION}

The ET and CE are two currently proposed third generation detectors. Due to the huge improvement in the sensitivity in the frequency band below $10 \mathrm{~Hz}$, the inband durations of the gravitational waves detected from
BNS mergers will be hours or even days long. Therefore the Earth's rotation will become important, leading to several effects that become relevant for such long in-band duration signals. The long in-band duration allows us to observe the signal from different positions along the detector trajectory as the earth rotates. This in turn leads to a time-dependent detector response during the signal and also causes the wave to be Doppler modulated.

Using the Fisher matrix and taking the earth's rotation into consideration, we have estimated the localization capabilities of the ET and CE individually and as a network for BNS sources at distances equal to 40,200,400,800, and $1600 \mathrm{Mpc}$ and for a population of BNS sources that is distributed uniformly in comoving volume. We have found that for BNS at 40 and $200 \mathrm{Mpc}$, the ET alone will be able to localize most of the signals to within $100 \mathrm{deg}^{2}$ with 90\% confidence. If we assume EM follow up observation is achievable for BNS whose associated $90 \%$ credible region is $\leq 100 \mathrm{deg}^{2}$, this means the ET alone will be able to provide support for multimessenger astronomy for BNS mergers within $200 \mathrm{Mpc}$. However, for distances $\geq 400 \mathrm{Mpc}$, localization from the ET alone will still be poor. This is consistent with the localization performance for a population of BNS distributed uniformly in comoving volume. Of the detectable sources, only $\sim 32 \%$ can be localized with $90 \%$ to within a region less than the size of the whole sky.

Combining the ET and CE can dramatically boost the performance in localization. Almost all the sources within $1600 \mathrm{Mpc}$ can be localized to within $100 \mathrm{deg}^{2}$ with 90\% confidence. In particular, the upper limit of the $90 \%$ credible region for the best localized $90 \%$ of the detectable sources at 40 and $200 \mathrm{Mpc}$ has reduced by $\sim 100$ times compared to using only the ET. Similar or greater improvements are seen for sources at greater distances. For a population of BNS uniformly distributed in the comoving volume, the improvement is equally impressive. The upper limit of the $90 \%$ credible region for the best localized $90 \%$ of the detectable sources as derived from the Fisher matrix shrinks from an area larger than the entire sky to $\sim 500 \mathrm{deg}^{2}$.

Regarding the ability to send event alerts prior to merger, the trend is similar. Using the ET alone, alerts for most BNSs within $200 \mathrm{Mpc}$ can be sent a few hours prior to merger, while for BNSs at $\geq 400 \mathrm{Mpc}$, a large fraction of sources do not meet our early warning criteria. Those which do meet the criteria do so at a time relatively close to merger $\left(\mathcal{O}(10)-\mathcal{O}\left(10^{2}\right)\right.$ minutes). A network with both the ET and CE substantially increases the number of signals at distances $\geq 400 \mathrm{Mpc}$ that meet the early warning criteria. This highlights the desirability and potential of such a network for BNS at relatively large distances. By turning on and off the Doppler effect in the simulation, we also established that the modulation of detector responses during the in-band duration is the main cause for improved localization. 


\section{ACKNOWLEDGMENTS}

We are grateful to Prof. Yanbei Chen for his help with the methodology of the paper, and to Prof. Steve Fairhurst for his constructive comments on the paper. We are thankful to Dr. Xilong Fan and Teng Zhang for constructive discussions of this work. We are also grateful for computational resources provided by
Cardiff University, and we are funded by an STFC grant supporting UK Involvement in the Operation of Advanced LIGO. This research is supported by The Scottish Universities Physics Alliance and Science and Technology Facilities Council. I. S. H., C. M., and M. H. are supported by the Science and Technology Research Council (Grant No. ST/L000946/1)
[1] B. P. Abbott, R. Abbott, T. D. Abbott, M. R. Abernathy, F. Acernese, K. Ackley, C. Adams, T. Adams, P. Addesso, R. X. Adhikari et al., Observation of Gravitational Waves from a Binary Black Hole Merger, Phys. Rev. Lett. 116, 061102 (2016).

[2] B. P. Abbott, R. Abbott, T. D. Abbott, M. R. Abernathy, F. Acernese, K. Ackley, C. Adams, T. Adams, P. Addesso, R. X. Adhikari et al., GW151226: Observation of Gravitational Waves from a 22-Solar-Mass Binary Black Hole Coalescence, Phys. Rev. Lett. 116, 241103 (2016).

[3] B. P. Abbott, R. Abbott, T. D. Abbott, F. Acernese, K. Ackley, C. Adams, T. Adams, P. Addesso, R. X. Adhikari, V. B. Adya et al., GW170104: Observation of a 50-SolarMass Binary Black Hole Coalescence at Redshift 0.2, Phys. Rev. Lett. 118, 221101 (2017).

[4] B. P. Abbott, R. Abbott, T. D. Abbott, F. Acernese, K. Ackley, C. Adams, T. Adams, P. Addesso, R. X. Adhikari, V. B. Adya et al., GW170608: Observation of a 19 solarmass binary black hole coalescence, Astrophys. J. Lett. 851, L35 (2017).

[5] B. P. Abbott, R. Abbott, T. D. Abbott, F. Acernese, K. Ackley, C. Adams, T. Adams, P. Addesso, R. X. Adhikari, V. B. Adya et al., GW170814: A Three-Detector Observation of Gravitational Waves from a Binary Black Hole Coalescence, Phys. Rev. Lett. 119, 141101 (2017).

[6] B. P. Abbott, R. Abbott, T. D. Abbott, F. Acernese, K. Ackley, C. Adams, T. Adams, P. Addesso, R. X. Adhikari, V. B. Adya et al., GW170817: Observation of Gravitational Waves from a Binary Neutron Star Inspiral, Phys. Rev. Lett. 119, 161101 (2017).

[7] B. P. Abbott, R. Abbott, T. D. Abbott, F. Acernese, K. Ackley, C. Adams, T. Adams, P. Addesso, R. X. Adhikari, V. B. Adya et al., Gravitational waves and gamma-rays from a binary neutron star merger: Gw170817 and grb 170817a, Astrophys. J. Lett. 848, L13 (2017).

[8] R. Margutti, E. Berger, W. Fong, C. Guidorzi, K. D. Alexander, B. D. Metzger, P. K. Blanchard, P. S. Cowperthwaite, R. Chornock, T. Eftekhari et al., The electromagnetic counterpart of the binary neutron star merger LIGO/Virgo GW170817. V. Rising X-ray emission from an off-axis jet, Astrophys. J. Lett. 848, L20 (2017).

[9] A. Goldstein, P. Veres, E. Burns, M. S. Briggs, R. Hamburg, D. Kocevski, C. A. Wilson-Hodge, R. D. Preece, S. Poolakkil, O. J. Roberts et al., An ordinary short gammaray burst with extraordinary implications: Fermi-gbm detection of grb 170817a, Astrophys. J. Lett. 848, L14 (2017).

[10] B. P. Abbott, R. Abbott, T. D. Abbott, F. Acernese, K. Ackley, C. Adams, T. Adams, P. Addesso, R. X. Adhikari, V.B. Adya et al., Multi-messenger observations of a binary neutron star merger, Astrophys. J. Lett. 848, L12 (2017).

[11] N. R. Tanvir, A. J. Levan, C. Gonzalez-Fernandez, O. Korobkin, I. Mandel, S. Rosswog, J. Hjorth, P. D’Avanzo, A. S. Fruchter, C. L. Fryer et al., The emergence of a Lanthanide-rich kilonova following the merger of two neutron stars, Astrophys. J. Lett. 848, L27 (2017).

[12] B. P. Abbott, R. Abbott, T. D. Abbott, M. R. Abernathy, F. Acernese, K. Ackley, C. Adams, T. Adams, P. Addesso, R. X. Adhikari et al., Prospects for observing and localizing gravitational-wave transients with advanced LIGO and advanced Virgo, Living Rev. Relativity 19, 1 (2016).

[13] B. S. Sathyaprakash, S. Fairhurst, B. F. Schutz, J. Veitch, S. Klimenko, D. Reitze, and S. Whitcomb, Scientific benefits of moving one of LIGO Hanford detectors to India, LIGO Report No. 1200219, 2012.

[14] Y. Aso, Y. Michimura, K. Somiya, M. Ando, O. Miyakawa, T. Sekiguchi, D. Tatsumi, and H. Yamamoto, Interferometer design of the KAGRA gravitational wave detector, Phys. Rev. D 88, 043007 (2013).

[15] B. P. Abbott, R. Abbott, T. D. Abbott, M. R. Abernathy, F. Acernese, K. Ackley, C. Adams, T. Adams, P. Addesso, R. X. Adhikari et al., All-sky search for short gravitationalwave bursts in the first Advanced LIGO run, Phys. Rev. D 95, 042003 (2017).

[16] T. Damour and A. Vilenkin, Gravitational radiation from cosmic (super)strings: Bursts, stochastic background, and observational windows, Phys. Rev. D 71, 063510 (2005).

[17] P. S. Cowperthwaite and E. Berger, A comprehensive study of detectability and contamination in deep rapid optical searches for gravitational wave counterparts, Astrophys. J. 814, 25 (2015).

[18] B. D. Metzger and E. Berger, What is the most promising electromagnetic counterpart of a neutron star binary merger?, Astrophys. J. 746, 48 (2012).

[19] N. R. Tanvir, A. J. Levan, A. S. Fruchter, J. Hjorth, R. A. Hounsell, K. Wiersema, and R. L. Tunnicliffe, A 'kilonova' associated with the short-duration $\gamma$-ray burst GRB 130603B, Nature (London) 500, 547 (2013). 
[20] E. Berger, W. Fong, and R. Chornock, An r-process kilonova associated with the short-hard GRB 130603B, Astrophys. J. Lett. 774, L23 (2013).

[21] E. Chassande-Mottin, M. Hendry, P. J. Sutton, and S. Márka, Multimessenger astronomy with the Einstein Telescope, Gen. Relativ. Gravit. 43, 437 (2011).

[22] J. S. Bloom, D. E. Holz, S. A. Hughes, and K. Menou, Coordinated science in the gravitational and electromagnetic skies. in astro2010, arXiv:0902.1527.

[23] J. Kanner, T. L. Huard, S. Márka, D. C. Murphy, J. Piscionere, M. Reed, and P. Shawhan, LOOC UP: Locating and observing optical counterparts to gravitational wave bursts, Classical Quantum Gravity 25, 184034 (2008).

[24] B.F. Schutz, Determining the Hubble constant from gravitational wave observations, Nature (London) 323, 310 (1986).

[25] B. S. Sathyaprakash, B. F. Schutz, and C. Van Den Broeck, Cosmography with the Einstein Telescope, Classical Quantum Gravity 27, 215006 (2010).

[26] B. P. Abbott, R. Abbott, T. D. Abbott, F. Acernese, K. Ackley, C. Adams, T. Adams, P. Addesso, R. X. Adhikari, V. B. Adya et al., A gravitational-wave standard siren measurement of the Hubble constant, Nature (London) 551, 85 (2017).

[27] S. Fairhurst, Source localization with an advanced gravitational wave detector network, Classical Quantum Gravity 28, 105021 (2011).

[28] S. Nissanke, J. Sievers, N. Dalal, and D. Holz, Localizing compact binary inspirals on the sky using ground-based gravitational wave interferometers, Astrophys. J. 739, 99 (2011).

[29] S. Nissanke, M. Kasliwal, and A. Georgieva, Identifying elusive electromagnetic counterparts to gravitational wave mergers: An end-to-end simulation, Astrophys. J. 767, 124 (2013).

[30] J. Veitch, I. Mandel, B. Aylott, B. Farr, V. Raymond, C. Rodriguez, M. van der Sluys, V. Kalogera, and A. Vecchio, Estimating parameters of coalescing compact binaries with proposed advanced detector networks, Phys. Rev. D 85, 104045 (2012).

[31] C. L. Rodriguez, B. Farr, V. Raymond, W. M. Farr, T. B. Littenberg, D. Fazi, and V. Kalogera, Basic parameter estimation of binary neutron star systems by the Advanced LIGO/Virgo Network, Astrophys. J. 784, 119 (2014).

[32] K. Cannon, R. Cariou, A. Chapman, M. Crispin-Ortuzar, N. Fotopoulos, M. Frei, C. Hanna, E. Kara, D. Keppel, L. Liao, S. Privitera, A. Searle, L. Singer, and A. Weinstein, Toward early-warning detection of gravitational waves from compact binary coalescence, Astrophys. J. 748, 136 (2012).

[33] H.-Y. Chen and D. E. Holz, Facilitating follow-up of LIGOVirgo events using rapid sky localization, Astrophys. J. (2015).

[34] J. Mills, V. Tiwari, and S. Fairhurst, Localization of binary mergers with gravitational-wave detectors of second and third generation, Phys. Rev. D 97, 104064 (2018).

[35] W. Zhao and L. Wen, Localization accuracy of compact binary coalescences detected by the third-generation gravitational-wave detectors and implication for cosmology, Phys. Rev. D 97, 064031 (2018).

[36] B. Sathyaprakash, M. Abernathy, F. Acernese, P. Ajith, B. Allen, P. Amaro-Seoane, N. Andersson, S. Aoudia, K. Arun, P. Astone et al., Scientific objectives of Einstein Telescope, Classical Quantum Gravity 29, 124013 (2012).

[37] LIGO Scientific Collabration, Ligo instrument science white paper, Report No. LIGO-T1700231-v2, 2017.

[38] T. Regimbau, T. Dent, W. Del Pozzo, S. Giampanis, T. G. F. Li, C. Robinson, C. Van Den Broeck, D. Meacher, C. Rodriguez, B.S. Sathyaprakash, and K. Wójcik, A mock data challenge for the Einstein gravitational-wave telescope, Phys. Rev. D 86, 122001 (2012).

[39] M. Punturo, M. Abernathy, F. Acernese, B. Allen, N. Andersson, K. Arun, F. Barone, B. Barr, M. Barsuglia, M. Beker et al., The Einstein Telescope: A third-generation gravitational wave observatory, Classical Quantum Gravity 27, 194002 (2010).

[40] M. Pitkin, S. Reid, S. Rowan, and J. Hough, Gravitational wave detection by interferometry (ground and space), Living Rev. Relativity 14, 5 (2011).

[41] S. Hild, M. Abernathy, F. Acernese, P. Amaro-Seoane, N. Andersson, K. Arun, F. Barone, B. Barr, M. Barsuglia, M. Beker et al., Sensitivity studies for third-generation gravitational wave observatories, Classical Quantum Gravity 28, 094013 (2011).

[42] S. Hild, S. Chelkowski, A. Freise, J. Franc, N. Morgado, R. Flaminio, and R. DeSalvo, A xylophone configuration for a third-generation gravitational wave detector, Classical Quantym Gravity 27, 015003 (2010).

[43] S. Hild, S. Chelkowski, and A. Freise, Pushing towards the ET sensitivity using 'conventional' technology, arXiv: 0810.0604.

[44] M. Bassan, Advanced Interferometers and the Search for Gravitational Waves: Lectures from the First VESF School on Advanced Detectors for Gravitational Waves (Springer, New York, 2014), Vol. 404.

[45] M. Vallisneri, Use and abuse of the Fisher information matrix in the assessment of gravitational-wave parameterestimation prospects, Phys. Rev. D 77, 042001 (2008).

[46] M. Zanolin, S. Vitale, and N. Makris, Application of asymptotic expansions for maximum likelihood estimators errors to gravitational waves from binary mergers: The single interferometer case, Phys. Rev. D 81, 124048 (2010).

[47] H.-S. Cho and C.-H. Lee, Application of the effective Fisher matrix to the frequency domain inspiral waveforms, Classical Quantum Gravity 31, 235009 (2014).

[48] B. P. Abbott, R. Abbott, T. D. Abbott, M. R. Abernathy, F. Acernese, K. Ackley, C. Adams, T. Adams, P. Addesso, R. X. Adhikari et al., Localization and broadband follow-up of the gravitational-wave transient GW150914, Astrophys. J. Lett. 826, L13 (2016).

[49] M. L. Chan, Y.-M. Hu, C. Messenger, M. Hendry, and I. S. Heng, Maximizing the detection probability of kilonovae associated with gravitational wave observations, Astrophys. J. 834, 84 (2017).

[50] S. Fairhurst, Triangulation of gravitational wave sources with a network of detectors, New J. Phys. 11, 123006 (2009). 
[51] S. Vitale, W. Del Pozzo, T. G. F. Li, C. Van Den Broeck, I. Mandel, B. Aylott, and J. Veitch, Effect of calibration errors on Bayesian parameter estimation for gravitational wave signals from inspiral binary systems in the advanced detectors era, Phys. Rev. D 85, 064034 (2012).
[52] L. Lindblom, Optimal calibration accuracy for gravitationalwave detectors, Phys. Rev. D 80, 042005 (2009).

[53] L. Lindblom, J. G. Baker, and B. J. Owen, Improved time-domain accuracy standards for model gravitational waveforms, Phys. Rev. D 82, 084020 (2010). 